\title{
Principled compassion
}

\begin{abstract}
Investigational drugs can save or extend lives, and seriously ill patients not able to take part in clinical trials should have access to such drugs whenever possible. In a climate of increased public pressure for this access-often termed compassionate use - five states in the US have passed so-called 'right to try' legislation. These laws are ill advised, as they are not likely to substantially increase access and have the potential to compromise the clinical trial system.
\end{abstract}

$\mathbf{U}$ nder rules first established in 1987 and then clarified and codified in August 2009, the US Food and Drug Administration (FDA) allows patients who have serious and life-threatening conditions access to investigational drugs. Under these rules, the patient seeking access must not be eligible for an existing clinical trial and must obtain both approval from an institutional review board and an agreement from the company developing the drug to provide it. The FDA then decides whether to approve the request, considering, among other factors, whether the potential benefit justifies the potential risks and whether expanded access would compromise the clinical development of the drug towards marketing approval.

According to the FDA, it approves virtually all the requests it receives, so that in practice, the major potential roadblock for a patient requesting a drug is persuading the company to provide it. Companies understandably prioritize their resources to support clinical trials and may be unwilling or unable to provide an investigational drug outside of a trial. For example, they may not have enough of the drug available or sufficient manpower to handle the logistics of providing the drug to the patient and communicating with the FDA. Companies may also worry that any adverse events that the patient experiences while taking the drug may derail its eventual approval, although no specific cases in which this occurred have surfaced.

Recently, the rise of social media has changed the landscape for drug companies. Social media campaigns mounted on the behalf of patients unable to obtain a potentially life-saving drug have put companies under tremendous pressure. For example, in March of this year, Josh Hardy, a seven-year-old boy, had a life-threatening adenovirus infection after a bone marrow transplant. He was initially denied access to an antiviral compound, brincidofovir, by its manufacturer, Chimerix, which was conducting a clinical trial of the compound for the prevention of cytomegalovirus infection in adults undergoing hematopoietic stem cell transplantation. Chimerix, a small biotechnology company, explained that it had limited manpower and financial resources, and could not respond to the large demand for compassionate access to the drug. After a social media campaign on behalf of Hardy was launched, the company was vilified, and its management received death threats. Caught in this media firestorm, the FDA and the drug company were able to design an open-label pilot trial of the drug for 20 patients, allowing Hardy to get the drug, to which he very quickly responded.

This pressure on companies and regulators for increased access to investigational drugs has exposed cracks in the system. Companies vary in their accessibility to patients asking for an investigational drug and in their preparedness to handle these requests, and they are left to make seemingly ad hoc decisions. It also does not seem fair that whether a patient gets a drug depends on his or her ability to wage an effective social media campaign.

Legislators are now getting involved. Right-to-try laws recently enacted by five states-including an Arizona ballot measure passed this month by a large majority of voters-allow patients with life-threatening conditions access to treatments that have successfully completed phase 1 safety testing and remove oversight by the FDA, with the goal of removing bureaucratic red tape. However, companies are still left to decide whether or not to provide the drug, and so these laws do not address the real roadblock to getting drugs to patients. By removing FDA oversight, these measures could put patients at risk and could compromise the clinical trial system. Why enroll in a clinical trial, where you might be given a placebo, if you can get the drug prescribed?

Although states are going in the wrong direction, legislative efforts at the federal level may be a step forward. As outlined in a recent white paper (http://freepdfhosting.com/4c864343e3.pdf), Representative Michael McCaul, a congressman from Texas, is planning legislation to increase the transparency of the compassionate-use process and to make this process easier for patients to navigate. The legislation would, for example, require companies to provide clear directions for how patients can request drugs and would also require them to provide explanations to patients when requests are denied. Companies would also have to report the number of drug requests to the FDA, which would then supply this information to Congress to inform policy making.

However, more needs to be done. Companies should be incentivized to increase drug availability, and the process by which companies and regulators make the hard choice of whether to provide a drug to a critically ill patient should be made as systematic and transparent as possible. Other ideas on the table are to provide safe harbor to companies to protect them from the negative consequences of adverse events that may occur in patients taking their drug in a compassionate-use setting and to set up an independent advisory group of the relevant stakeholders-industry, patient advocates and regulators-that could help companies come to principled decisions and defuse the perception that they unfairly withhold lifesaving therapies.

As the momentum of right-to-try legislation demonstrates, the public increasingly demands access to the compassionate use of investigational treatments, and companies and regulators need to adapt to this new reality. But these life-and-death decisions can't be left solely to the company that makes the drug. The FDA must maintain oversight and must defend the overarching goals of the clinical trial system - that safe and effective drugs get to as many people as possible as quickly as possible. 